\title{
Author Correction: Evolution of microscopic heterogeneity and dynamics in choline chloride- based deep eutectic solvents
}

\author{
Stephanie Spittle, Derrick Poe, Brian Doherty, Charles Kolodziej (1D, Luke Heroux (D), Md Ashraful Haque, \\ Henry Squire (1D, Tyler Cosby, Yong Zhang, Carla Fraenza, Sahana Bhattacharyya, Madhusudan Tyagi (D), \\ Jing Peng, Ramez A. Elgammal, Thomas Zawodzinski, Mark Tuckerman (1), Steve Greenbaum (D), Burcu Gurkan, \\ Clemens Burda, Mark Dadmun, Edward J. Maginn (1) \& Joshua Sangoro (1)
}

Correction to: Nature Communications https://doi.org/10.1038/s41467-021-27842-z, published online 11 January 2022.

The original version of this Article, and the original version of the Supplementary Information associated with this Article, omitted from the author list the $19^{\text {th }}$ author Clemens Burda, who is from the Department of Chemistry, Case Western Reserve University, Cleveland, OH, 44106, USA. The text 'C.K. and C.B. provided the femtosecond transient-absorption spectroscopy experiments and analysis' was already present in the Author Contributions. The author list has been corrected in both the PDF and HTML versions of the Article, and the HTML has been updated to include a corrected version of the Supplementary Information.

Published online: 22 February 2022

\section{Additional information}

Supplementary information The online version contains supplementary material available at https://doi.org/10.1038/s41467-022-28671-4. 